\title{
The SNARE Proteins SNAP25 and Synaptobrevin Are Involved in Endocytosis at Hippocampal Synapses
}

\author{
Zhen Zhang, ${ }^{1}$ Dongsheng Wang, ${ }^{1}$ Tao Sun, ${ }^{1}$ Jianhua Xu, ${ }^{2}$ Hsueh-Cheng Chiang, ${ }^{1}$ Wonchul Shin, ${ }^{1}$ and Ling-Gang Wu ${ }^{1}$ \\ ${ }^{1}$ National Institute of Neurological Disorders and Stroke, Bethesda, Maryland 20892, and ${ }^{2}$ Institute of Molecular Medicine and Genetics and Department of \\ Neurology, Georgia Health Sciences University, Augusta, Georgia 30912
}

\begin{abstract}
SNAP25, an essential component of the soluble NSF ( $N$-ethylmaleimide-sensitive factor) attachment protein receptor (SNARE) complex that mediates exocytosis, is not considered to play a role in endocytosis, which couples to exocytosis by retrieving a similar amount of exocytosed vesicles. By knocking down SNAP25 and imaging slow endocytosis at a conventional synapse, the rat cultured hippocampal synapse, we found that SNAP25 is involved in slow, clathrin-dependent endocytosis. With similar techniques, we found that not only SNAP25, but also synaptobrevin is involved in slow endocytosis. These results provide the first evidence showing the dual role of SNAP25 and synaptobrevin in both exocytosis and slow endocytosis at conventional synapses. Such a dual role may contribute to mediate the coupling between exocytosis and clathrin-dependent endocytosis at conventional synapses, a mechanism critical for the maintenance of synaptic transmission and the normal structure of nerve terminals.
\end{abstract}

\section{Introduction}

Vesicle exocytosis is catalyzed by the SNARE complex, composed of synaptobrevin at the vesicle membrane and SNAP25 and syntaxin at the plasma membrane (Sudhof, 2004; Jackson and Chapman, 2008). After exocytosis, endocytosis retrieves fused vesicle membrane and proteins (Sudhof, 2004). The most common form of endocytosis is slow endocytosis, which occurs over tens of seconds (Wu et al., 2007). Slow endocytosis involves many classical endocytic proteins, such as dynamin, clathrin, AP2, and auxilin, which are different from the SNARE proteins (Dittman and Ryan, 2009).

Because of the overwhelming role of SNARE proteins in exocytosis and a seemingly clear difference between exocytic and endocytic protein machineries, only three pioneering studies have examined the role of SNARE proteins in endocytosis. One study shows that SNAP25 knock-out does not affect FM dye uptake into hippocampal boutons after sucrose application, leading to the suggestion that SNAP25 is not involved in endocytosis (Bronk et al., 2007). A knock-out study supports a role of synaptobrevin in rapid endocytosis in hippocampal synapses (Deák et al., 2004). However, this suggestion remains to be verified, because it remains debated whether the technique used (FM dye imaging) in this study can detect rapid endocytosis, and whether rapid endocytosis exists at hippocampal synapses ( $\mathrm{He}$ and $\mathrm{Wu}$, 2007; Granseth et al., 2009; Zhang et al., 2009). A recent study

\footnotetext{
Received Jan. 22, 2013; revised April 17, 2013; accepted April 20, 2013.

Author contributions:Z.Z., D.W., T.S., J.X., H.-C.C., W.S., and L.-G.W. designed research; Z.Z. and D.W. performed research; Z.Z. and D.W. analyzed data; Z.Z. and L.-G.W. wrote the paper.

This work was supported by the NINDS Intramural Research Program. We thank Edaeni Hamid for comments on this manuscript, and Dr. Yongling Zhu (Northwestern University, Chicago) for providing synaptophysin-pHluorin2X plasmid.

Correspondence should be addressed to Ling-Gang Wu, National Institute of Neurological Disorders and Stroke, 35 Convent Drive, Building 35, Room 2B-1012, Bethesda, MD 20892. E-mail: wul@ninds.nih.gov.

DOI:10.1523/JNEUROSCI.0301-13.2013

Copyright $\odot 2013$ the authors $\quad 0270-6474 / 13 / 339169-07 \$ 15.00 / 0$
}

showed that tetanus toxin blocks slow endocytosis at a giant nerve terminal, the calyx of Held, suggesting the involvement of synaptobrevin in slow clathrin-dependent endocytosis (Hosoi et al., 2009). Together, synaptobrevin is considered the only SNARE protein involved in endocytosis, which may mediate the coupling between exocytosis and slow clathrin-dependent endocytosis (Hosoi et al., 2009). Three questions remain unresolved. First, tetanus toxin cleaves synaptobrevin into fragments. Whether the block of endocytosis by tetanus toxin is due to a side effect caused by fragments of synaptobrevin remains unclear. Additional, independent evidence is needed to fully consolidate the suggestion that synaptobrevin is involved in slow endocytosis. Second, it is unclear whether the results obtained at the giant calyx-type synapse apply to conventional small synapses. Third, whether the vesicular SNARE, synaptobrevin, is the only SNARE involved in endocytosis remains unclear.

In the present work we addressed these three issues by knocking down SNAP25 and synaptobrevin at a conventional synapse, the cultured hippocampal synapse. We found that not only synaptobrevin, but also SNAP25, is involved in slow, clathrin-dependent endocytosis. This finding calls for correction of the previous suggestion that SNAP25 is not involved in endocytosis at hippocampal synapses. It further elucidates the previous finding on the role of synaptobrevin in endocytosis, and extends this finding from giant synapses to conventional small synapses. Thus, not only synaptobrevin, but also SNAP25, plays an important role in endocytosis, and may contribute to the coupling between exocytosis and slow clathrin-dependent endocytosis at synapses.

\section{Materials and Methods}

Hippocampal culture, fluorescence imaging, and statistics. Hippocampal culture, stimulation, and fluorescence imaging were performed as described previously (Sun et al., 2010). Briefly, hippocampal CA1-CA3 regions from $\mathrm{P} 0-2$ Sprague Dawley rats of either sex were dissociated and plated onto poly-D-lysine-coated glass coverslips and incubated $\left(37^{\circ} \mathrm{C}\right.$, 
$5 \% \mathrm{CO}_{2}$ ) in a medium consisting of MEM, $0.5 \%$ glucose, $0.1 \mathrm{~g} / \mathrm{L}$ bovine transferrin, $0.3 \mathrm{~g} / \mathrm{L}$-glutamine, $10 \%$ fetal bovine serum, $2 \% \mathrm{~B}-27$, and 3 $\mu \mathrm{M}$ cytosine $\beta$-D-arabinofuranoside. Six to eight days after plating, calcium-phosphate-mediated gene transfer was used to transfect neurons with synaptophysin-pHluroin2X (SypH2X), and/or plasmids containing SNAP25 or synaptobrevin shRNA, and/or the respective shRNA-resistant plasmids. After transfection, neurons were incubated for 6-8 d before use. All reagents were from Sigma if not mentioned.

Action potential was evoked by a $1 \mathrm{~ms}$ pulse $(20 \mathrm{~mA})$ through a platinum electrode. The bath solution $\left(\sim 22-24^{\circ} \mathrm{C}\right)$ contained the following (in mM): $119 \mathrm{NaCl}, 2.5 \mathrm{KCl}, 2 \mathrm{CaCl}_{2}, 2 \mathrm{MgCl}_{2}, 25$ HEPES (buffered to $\mathrm{pH}$ 7.4), 30 glucose, 0.01 6-cyano-7-nitroquinoxaline-2, 3-dione (CNQX), and 0.05 D, L-2-amino-5-phosphonovaleric acid (AP-5).

SypH2X or fluo 2 images were acquired at $1 \mathrm{~Hz}$ using the Zeiss LSM510 META confocal microscope (with a $40 \times, 1.3$ NA oil-immersion objective), and analyzed with Zeiss LSM510 software. All boutons that showed a fluorescence increase during stimulation were analyzed (region of interest: $2 \times 2 \mu \mathrm{m}$ ). Each data group was obtained from at least three batches of cultures. Fluo 2 was excited at $488 \mathrm{~nm}$, and its fluorescence at $505-530 \mathrm{~nm}$ wavelength was collected.

The statistical test was an $t$ test. Means are presented as \pm SEM.

Plasmids, knockdown, and immunostaining. The sequence of the shRNA against rat SNAP25 in the pRFP-C-RS plasmid (Origene Technologies, Cat. \#: TF711123) was AAGATGCTGGCATCAGGACTTTG GTTATG. The scrambled sequence was GCACTACCAGAGCTAACT CAGATAGTACT (same for synaptobrevin shRNA). The RFP in the plasmid was cutoff using Pme I and BglII. Mutated human SNAP25 cDNA (corresponding targeted sequence: AAGATGCAGGTATTAGAACATT GGTTATG) was used as a shRNA-resistant sequence to rescue SNAP25 expression after knockdown.

The sequence of the shRNA against rat synaptobrevin in the pRFPC-RS plasmid (Origene Technologies, Cat. \#: TF709295) was GAT CATCTTGGGAGTGATTTGCGCCATCA. The RFP in the plasmid was cutoff using Pme I and BglII. Mutated human synaptobrevin cDNA (corresponding targeted sequence: GATCATATTAGGTGTCATATGCGC CATCA) was used as a shRNA-resistant sequence to rescue synaptobrevin expression after knockdown.

For immunostaining, neurons were fixed with $4 \%$ paraformaldehyde, permeabilized with $0.3 \%$ Triton X-100, and subsequently incubated with the primary and secondary antibodies. The primary antibodies were diluted in PBS containing 10\% donkey serum and incubated with cells for $1 \mathrm{~h}$ at room temperature. After several rinses in PBS, cells were incubated with fluorescence-conjugated donkey anti-sheep, anti-rabbit, or antimouse IgG (1:200, Jackson ImmunoResearch Lab) for $2 \mathrm{~h}$ at room temperature. Primary antibodies included sheep anti-GFP (1:300, Thermo Scientific), mouse anti-synaptobrevin, and anti-SNAP25 (1:800, Synaptic Systems), mouse anti-clathrin heavy chain (CLTC) (1:1000, Pierce), rabbit anti-dynamin (1:100, Santa Cruz Biotechnology), rabbit antiendophilin (1:200, Invitrogen), mouse anti-AP2 (1:100, Thermo Scientific), rabbit anti-RFP (1:400, Abcam), and goat anti-synapsin (1:100, Santa Cruz Biotechnology).

PC12 cells. For PC12 cell transfection, we used electroporation (Amaxa nucleofector II device, Lonza Walkersville, program U-029) and the Cell Line Nucleofector Kit $\mathrm{V}$ (Amaxa) with $2 \mu \mathrm{g}$ of plasmids. The transfection efficiency is $\sim 90 \%$ as detected by red fluorescence protein (RFP) expression. Cells were collected for Western blot analysis $3 \mathrm{~d}$ after transfection.

For Western blot, cell lysates were prepared in the modified RIPA buffer including protease inhibitors. Equal protein amounts were analyzed by SDS-PAGE and immunoblotting using antibodies against synaptobrevin (1:10,000, Synaptic Systems), SNAP25 (1:500, Synaptic Systems), mouse anti-clathrin heavy chain (CLTC; 1:1000, Pierce), rabbit anti-dynamin (1:100, Santa Cruz Biotechnology), rabbit antiendophilin (1:200, Invitrogen), mouse anti-AP2 (1:200, Thermo Scientific), and actin (internal control, 1: 400, Abcam).

\section{Results}

SNAP25 knockdown inhibits endocytosis

A SNAP25 shRNA was selected because it reduced SNAP25 expression to $14 \pm 6 \%$ of control (Western blot, $n=3, p<0.01$;
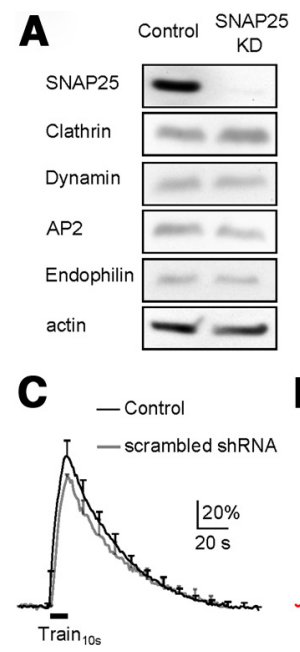

D

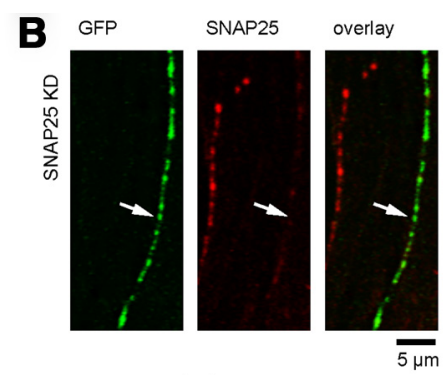

$\mathbf{F}$
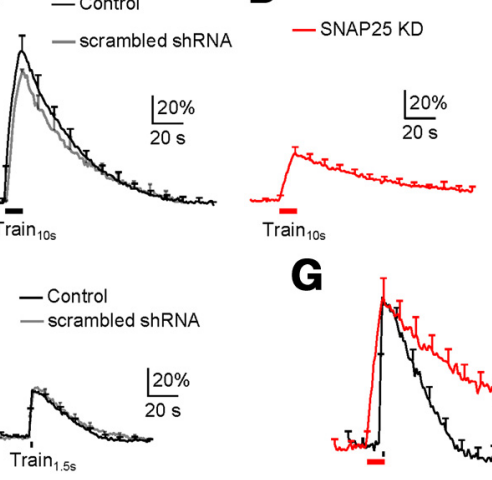

E

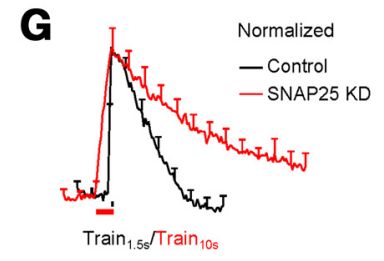

Figure 1. SNAP25 knockdown inhibits endocytosis. $\boldsymbol{A}$, Western blot of SNAP25, clathrin, dynamin, AP2, endophilin, and actin from PC12 cells in control (left) and in cells transfected with SNAP25 shRNA (KD, right). $\boldsymbol{B}$, Immunostaining of SypH2X (antibody against GFP, left, green) and SNAP25 (middle, red) at neuronal branches with (arrow, green staining) or without (no green staining) transfection of SypH2X and SNAP25 shRNA (right: left and middle panels superimposed). C, The SypH2X signal induced by Train ${ }_{10 \text { s }}$ at control boutons transfected with SypH2X (black, $n=6$ experiments) or with SypH2X and scrambled shRNA (gray, $n=4$ ). Data are expressed as the percentage change over the baseline intensity, and plotted as mean + SEM (every $10 \mathrm{~s}$, applies to all similar plots). D. The SypH2X signal induced by $\operatorname{Train}_{10 \mathrm{~s}}$ at boutons transfected with SypH2X and SNAP25 shRNA ( $n=12$ experiments, SNAP25 KD). $\boldsymbol{E}$, Traces in $\boldsymbol{C}$ (black only) and $\boldsymbol{D}$ (red) are normalized to the peak amplitude and superimposed. $\boldsymbol{F}$, The SypH $2 X$ signal induced by $\operatorname{Train}_{1.5 \mathrm{~s}}$ at control boutons transfected with only SypH2X (black, $n=7$ experiments) or with SypH2X and scrambled shRNA (gray, $n=7$ ). $\boldsymbol{G}$, Traces in $\boldsymbol{F}$ (black only) and $\boldsymbol{D}$ (red) are normalized to the same amplitude and superimposed.

Fig. 1A), but did not affect the expression of endocytic proteins, including clathrin heavy chain, dynamin, adaptor protein 2 (AP2), and endophilin in PC12 cells ( $n=3$ for each protein, $p>$ 0.05, Fig. $1 A$ ). At hippocampal cultures, we co-transfected this shRNA with SypH2X, the latter of which was used to detect exoendocytosis (Zhu et al., 2009) and to recognize transfected neurons. Co-transfection reduced SNAP2 5 expression in boutons, as detected with antibody staining, to $20 \pm 3 \%(n=447$ boutons from 3 transfections, $p<0.01$ ) of that in neighboring untransfected boutons (Fig. $1 B$ ).

To determine how SNAP25 knockdown affects endocytosis, we compared SypH2X fluorescence in neurons transfected with SypH2X alone (control) or both SypH2X and SNAP2 5 shRNA. In neurons transfected with SypH2X (control), a 10 s train, extracellular stimulation $(1 \mathrm{~ms}, 20 \mathrm{~mA})$ at $20 \mathrm{~Hz}\left(\right.$ Train $\left._{10 \mathrm{~s}}\right)$ induced a fluorescence increase $(\Delta F)$ of $97 \pm 11 \%$ (of the baseline intensity, $n=6$; Fig. 1C). The fluorescence increase was followed by a mono-exponential decay with a time constant $(\tau)$ of $38.2 \pm 2.4 \mathrm{~s}$ ( $n=6$, Fig. $1 C$ ), reflecting clathrin-dependent endocytosis (Granseth et al., 2006). Similar results were obtained in neurons co-transfected with scrambled shRNA and SypH2X (Fig. 1C, gray; $n=4)$.

In neurons co-transfected with SNAP2 5 shRNA and SypH2X, Train $_{10 \text { s }}$ induced a $\Delta F$ of $31 \pm 4 \%$, followed by a mono- 
exponential decay with a $\tau$ of $70.8 \pm 9.7 \mathrm{~s}(n=12)$ significantly slower than control $(38.2 \pm 2.4 \mathrm{~s}, p<0.05$; Fig. $1 D-E)$. Since the baseline fluorescence intensity was similar between control and SNAP25 shRNA-transfected boutons (data not shown), the $\Delta F$ in transfected boutons $(31 \pm 4 \%)$ was clearly smaller than that $(97 \pm 11 \%)$ in control boutons, consistent with the role of SNAP25 in exocytosis.

The prolonged endocytosis $\tau$ was not caused by the $\Delta F$ decrease for two reasons. First, in control, a $100 \mathrm{~Hz}$ train for $10 \mathrm{~s}$ induces a larger $\Delta F(\sim 2$ times larger $)$ and a longer endocytosis $\tau$ than those induced by $\operatorname{Train}_{10 \mathrm{~s}}$, whereas a $20 \mathrm{~Hz}$ train for $2 \mathrm{~s}$ induced a smaller $\Delta F(\sim 2$ times smaller $)$ and a faster endocytosis $\tau$ than those induced by $\operatorname{Train}_{10 \text { s }}$ (Sun et al., 2010). Thus, endocytosis $\tau$ increases only when the exocytosis amount increases to some extent that might saturate the endocytic capacity (Sankaranarayanan and Ryan, 2001). This phenomenon was not only observed at hippocampal synapses, but also at other synapses like neuromuscular junctions and calyx-type synapses (Wu and Betz, 1996; Sankaranarayanan and Ryan, 2001; Sun et al., 2002, 2010; Granseth et al., 2006; Renden and von Gersdorff, 2007; Balaji et al., 2008). Second, to verify this phenomenon in the present work, we applied a $1.5 \mathrm{~s}$ train at $20 \mathrm{~Hz}\left(\right.$ Train $\left._{1.5 \mathrm{~s}}\right)$ in control cultures to induce a $\Delta F(30 \pm 3 \%, n=7$; Fig. $1 F)$ similar to that $(31.4 \pm 3.8 \%)$ induced by Train $_{10 \text { s }}$ in SNAP25-knockdown neurons. This stimulus induced a fluorescence decay $\tau(29.5 \pm 4.2 \mathrm{~s}$, $n=7$; Fig. $1 F$ ) faster than that induced by Train ${ }_{10 \text { s }}$ in control or in SNAP25-knockdown neurons (Fig. 1G). Similarly fast decay was observed in cultures co-transfected with scrambled shRNA and SypH $2 X$ ( $n=7$; Fig. $1 F$, gray). We conclude that the prolonged endocytosis $\tau$ is not due to the reduced exocytosis.

To determine whether SNAP25 shRNA is specific to SNAP25, we co-transfected SNAP25 shRNA and a shRNA-resistant SNAP25 in PC12 cells, which increased SNAP25 expression to $238 \pm 21 \%$ of control $(n=3$, Fig. $2 A)$. We used PC1 2 cells here because in this cell line where transfection efficiency was high ( $\sim 90 \%$ ), we could perform Western blot to verify that the shRNA-resistant SNAP25 is resistant to SNAP25 shRNA. At hippocampal cultures where the transfection efficiency was much lower, we had to use immunostaining for the verification purpose. In hippocampal boutons transfected triply with SypH2X, SNAP25 shRNA, and shRNA-resistant SNAP25, SNAP25 was over rescued to $166 \pm 25 \%$ ( $n=420$ boutons, 3 transfections $)$ of that in un-transfected boutons (Fig. $2 B$ ). With triple transfection (SNAP25 rescue), Train ${ }_{10 \text { s }}$ induced a $\Delta F(92.5 \pm 11.1 \%, n=8$ experiments) similar to control (SypH2X transfection alone, $p>$ 0.05 ; Fig. $2 C$ ). The $\Delta F$ was followed by a mono-exponential decay $(35.7 \pm 7.1 \mathrm{~s}, n=8)$ similar to control $(38.2 \pm 2.4 \mathrm{~s}, n=12 ; p>$ 0.05; Fig. 2C). We conclude that SNAP25 shRNA inhibits endocytosis by knocking down SNAP25, but not by off-target shRNA effects.

To determine whether SNAP25 knockdown affects endocytic protein expression, we performed antibody staining of clathrin, dynamin, AP2, and endophilin in cultures co-transfected with SNAP25 shRNA and SypH2X or with scrambled shRNA and SypH2X (Fig. 2D). Transfected boutons were recognized with an antibody against green fluorescent protein (GFP), which also recognizes SypH2X (Fig. 2D). The immunostaining intensity for each of the four proteins in boutons co-transfected with SNAP25 shRNA and SypH2X was similar to that in boutons transfected with scrambled shRNA and SypH2X ( $p>0.05$; Fig. 2D-E, experimental numbers are specified in legends), or that in untransfected boutons (data not shown), indicating that SNAP25 shRNA does not reduce expression of major endocytic proteins.
Calcium influx speeds up endocytosis (Sankaranarayanan and Ryan, 2001; Balaji et al., 2008; Sun et al., 2010, but see Leitz and Kavalali, 2011). To determine whether SNAP25 knockdown reduces calcium influx, we loaded the boutons with fluo2 (5 $\mu \mathrm{M}$ fluo2-AM, 30 min incubation, then washout) in cultures transfected with both SNAP25 shRNA and synaptophysin-RFP. Train $_{10 \text { s }}$ induced fluo2 fluorescent spots, some of which were colocalized with synaptophysin-RFP spots (transfected boutons; Fig. $2 F$, red square), and some that were not (un-transfected boutons; Fig. $2 F$, green square). We compared fluo 2 fluorescence signal taken from the same image screen so that all controllable conditions, including fluo2-AM exposure time, fluorescence excitation, and emission settings, were identical. We found that the baseline, peak increase, and percentage increase of fluo 2 spots that were colocalized with synaptophysin-RFP spots (transfected boutons) were similar to those fluo2 spots that were not colocalized with RFP spots (un-transfected boutons, $n=6$ experiments, $p>0.05$; Fig. $2 F, G$ ). We also monitored fluo2 fluorescence increase at synaptophysin-RFP spots transfected with only synaptophysin-RFP (no SNAP25 knockdown, $n=8$ experiments, data not shown). The increase was also similar to that at synaptophysin-RFP spots transfected with synaptophysin-RFP and SNAP25 shRNA. In these experiments, synaptophysin-RFP spots were taken as boutons, because $94 \%$ of these spots (110 spots) as recognized with an antibody against RFP were colocalized with antibody staining against synapsin, a synaptic bouton marker (Fig. 2H) (Prodanov et al., 2006). Furthermore, fluo2 fluorescence increase in synaptophysin-RFP spots was much higher than in areas between the spots (Fig. $2 F$ ), consistent with localization of voltage-dependent calcium channels at boutons (Holderith et al., 2012; Sheng et al., 2012).

In summary, inhibition of endocytosis by SNAP25 shRNA was not due to reduction of exocytosis amount, endocytic proteins, or calcium influx or off-target shRNA effects. It was caused by SNAP2 5 knockdown, suggesting the involvement of SNAP25 in endocytosis.

\section{Synaptobrevin knockdown inhibits endocytosis}

A synaptobrevin shRNA knocked down synaptobrevin to $12 \pm$ $3 \%(n=4)$ of control without affecting endocytic proteins, including clathrin heavy chain, dynamin, AP2, and endophilin, in PC12 cells (Fig. $3 A ; n=3, p>0.05$ ). We therefore selected this shRNA for knockdown experiments at hippocampal cultures. In hippocampal boutons, co-transfection of synaptobrevin shRNA and SypH2X reduced synaptobrevin to $14 \pm 2 \%(n=247$ boutons from 3 transfections, $p<0.01$ ) of that in neighboring untransfected boutons, as detected with antibody staining (Fig. $3 B$ ). In synaptobrevin-knockdown neurons, Train 10 s induced a $\Delta F$ of $29 \pm 1 \%$ (of the baseline intensity, $n=10$ experiments), followed by a mono-exponential decay with a $\tau$ of $65.1 \pm 7.7 \mathrm{~s}(n=10$, Fig. $3 D)$, which was much slower than that induced by either Train ${ }_{10 \text { s }}$ $(38.2 \pm 2.4 \mathrm{~s}, n=6$; Fig. $3 C, E)$ or $\operatorname{Train}_{1.5 \mathrm{~s}}(29.5 \pm 4.2 \mathrm{~s}, n=7$; Fig. $3 F, G)$ in control $(p<0.05)$. The prolonged $\tau$ was not due to reduction of $\Delta F$, because in control conditions, Train ${ }_{1.5 s}$, which induced similar $\Delta F(30 \pm 3 \%$, Fig. $3 F)$, did not induce a prolonged fluorescence decay (Fig. $3 G$ ).

Co-transfection of a shRNA-resistant synaptobrevin with synaptobrevin shRNA rescued synaptobrevin expression to $179 \pm$ $37 \%$ of control $(n=4)$ in PC12 cells (Western blot, $n=4$, Fig. $4 A$ ). Triple-transfection of shRNA-resistant synaptobrevin, synaptobrevin shRNA, and SypH2X rescued synaptobrevin in transfected boutons to $132 \pm 30 \%$ ( $n=348$ boutons $)$ of that in neighboring un-transfected boutons (3 transfections, detected 
A

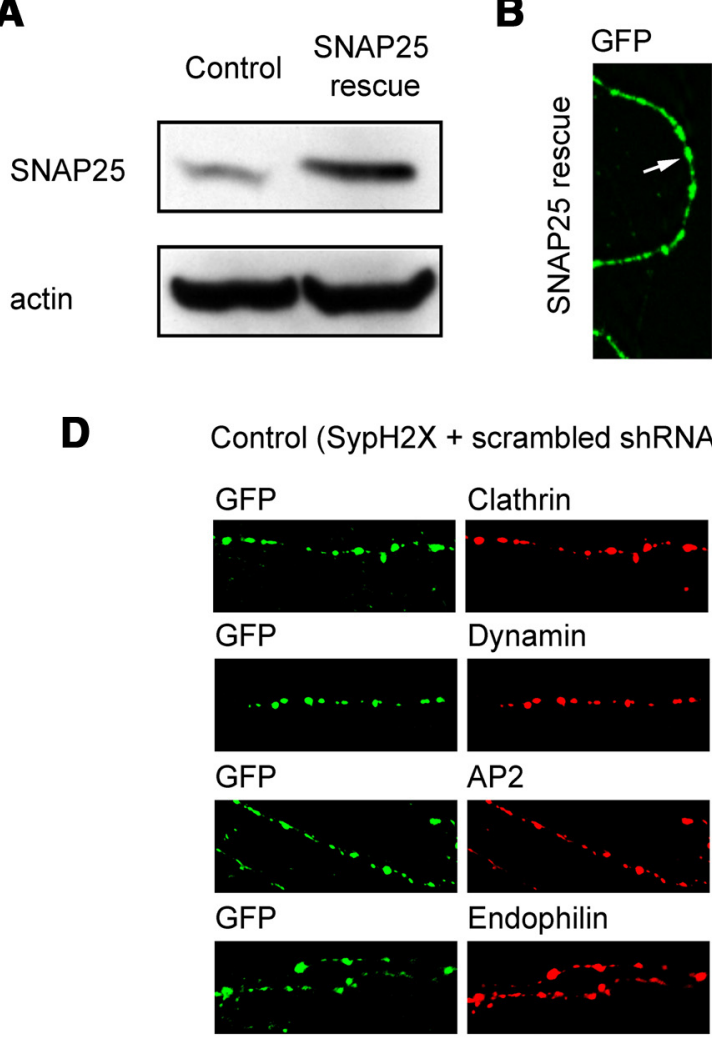

E

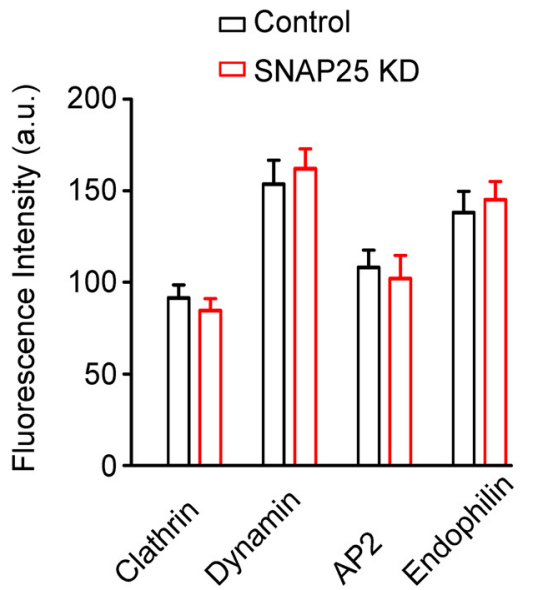

- Control

G

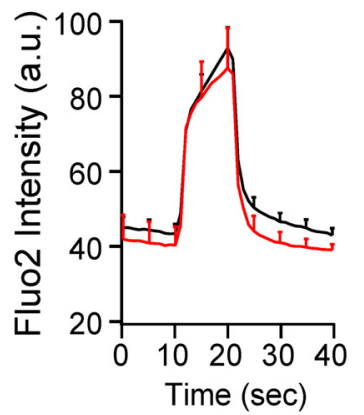

B

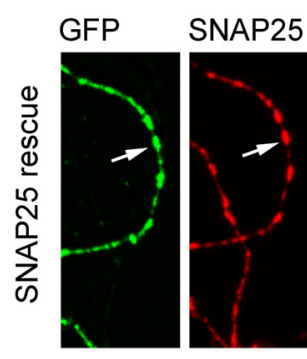

C

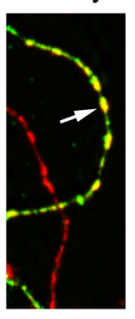

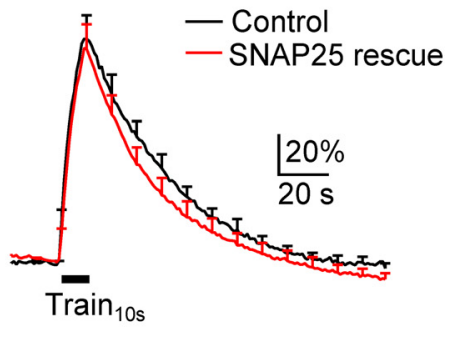

SNAP25 KD (SypH2X + shRNA)

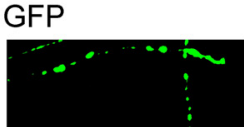

Clathrin
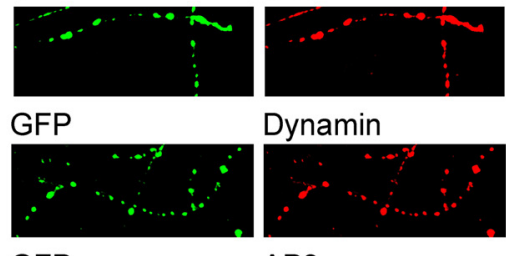

Dynamin

GFP
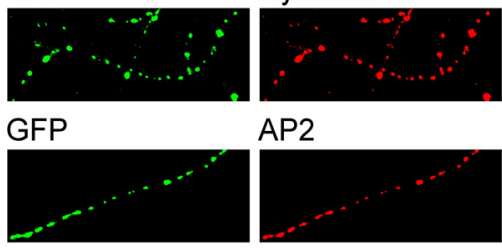

AP2
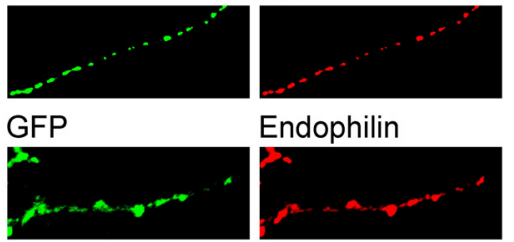

Endophilin

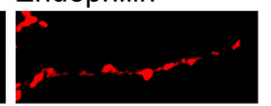

$\overline{5 \mu \mathrm{m}}$

$\mathbf{F}$

\section{SNAP25 KD+ SYP-RFP}

Fluo2

overlay
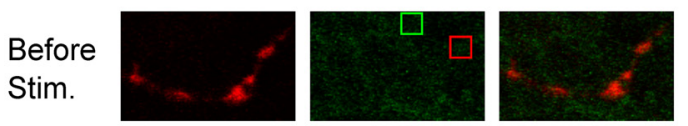

During

Stim.
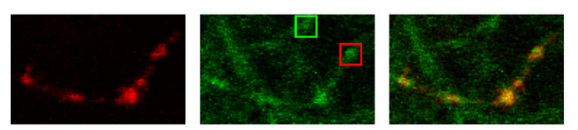

After

Stim.
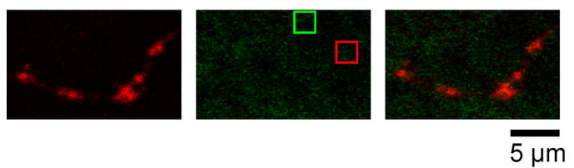

H

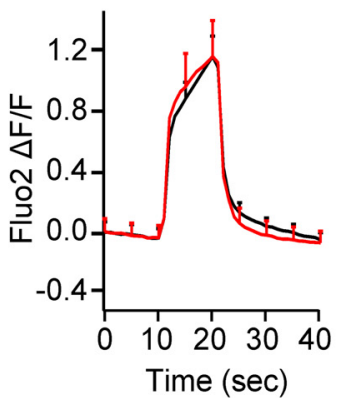

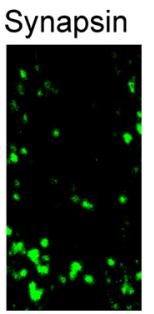

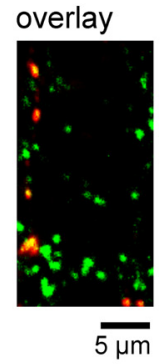

Figure 2. SNAP25 knockdown is specific. $\boldsymbol{A}$, Western blot of SNAP25 and actin from PC12 cells in control and in cells transfected with SNAP25 shRNA and shRNA-resistant SNAP25 (SNAP25 rescue). B, Immunostaining of SypH2X (antibody against GFP, left, green) and SNAP25 (middle, red) at neuronal branches with (arrow, green) or without (no green staining) triple-transfection of SypH2X, SNAP25 shRNA, and shNRA-resistant SNAP25 (SNAP25 rescue). Right, Left and middle panels superimposed. C, The SypH2X signal induced by Train ${ }_{10 \text { s }}$ at boutons transfected with only SypH2X (black, control, $n=6$ experiments) or with SypH2X, SNAP25 shRNA, and shNRA-resistant SNAP25 (SNAP25 rescue, red, $n=8$ experiments). D, Samples of (Figure legend continues.) 


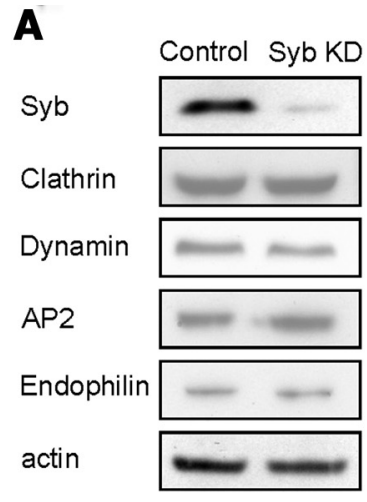

B
C

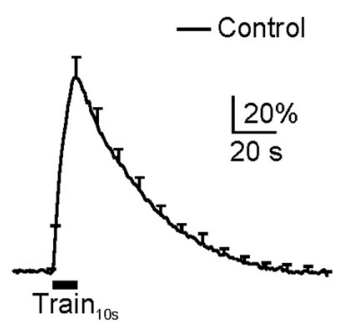

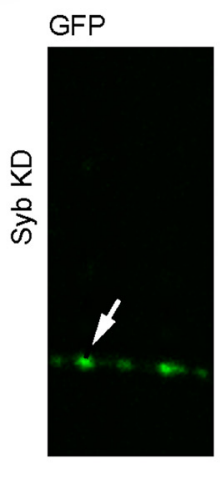

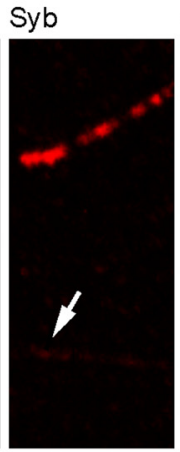

D

E
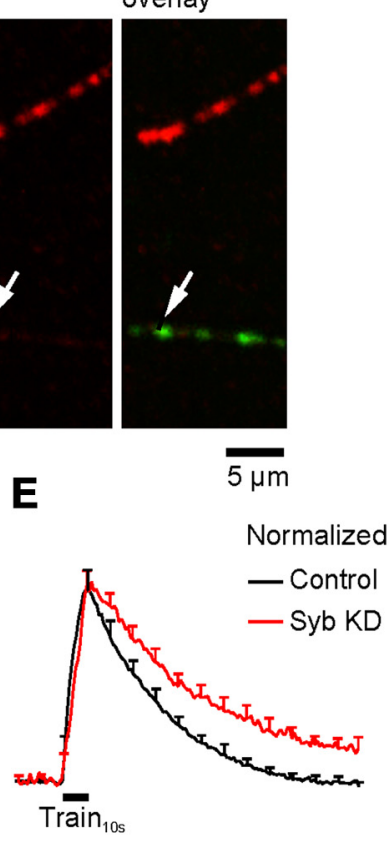

$\mathbf{F}$

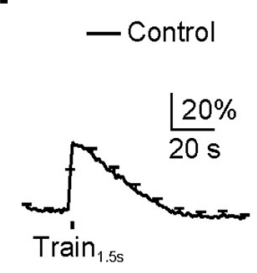

G

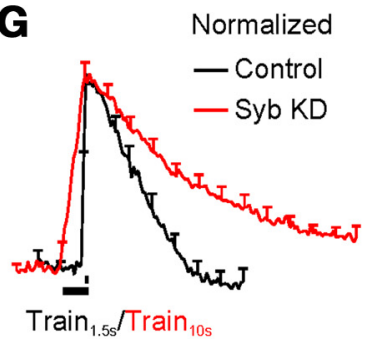

Figure 3. Synaptobrevin knockdown inhibits endocytosis. The arrangements in $\boldsymbol{A}-\mathbf{G}$ are the same as those in Figure $1 A-G$, respectively, except that SNAP25 in all legends and panels is replaced with synaptobrevin (or Syb). C, The trace is the same as the black trace in Figure 1C. $\boldsymbol{D}, n=10$ experiments from boutons transfected with SypH2X and Syb shRNA (Syb KD). $\boldsymbol{F}$, The trace is the same as the black trace in Figure 1F.

\footnotetext{
$\leftarrow$

(Figure legend continued.) immunostaining against GFP (green, recognizing SypH2X, first and third columns), clathrin, dynamin, AP2, and endophilin (red, second and fourth columns) at boutons transfected with scrambled shRNA and SypH2X (first and second columns, control) or with SNAP25 shRNA and SypH2X (third and fourth columns, SNAP25 KD). $\boldsymbol{E}$, The immunostaining intensity of clathrin, dynamin, AP2, and endophilin at boutons transfected with scrambled shRNA and SypH2X (control, black) or with SNAP25 shRNA and SypH2X (SNAP25 KD, red). Each group was taken from 2 transfections (clathrin: control, $n=126$ boutons; KD, $n=97$ boutons; dynamin: control, $n=97$ boutons; KD, $n=108$ boutons; AP2: control, $n=84$ boutons; KD, $n=86$ boutons; endophilin: control, $n=91$ boutons; KD, $n=95$ boutons). Control and knockdown were not statistically significant for each protein. $\boldsymbol{F}$, Sampled images showing fluo2 signal (calcium indicator, middle column, green) in neuronal branches with or without transfection of SNAP25 shRNA and synaptophysin-RFP (Syp-RFP, left column) at $10 \mathrm{~s}$ before (top), during (middle), and 20 s after (bottom) Train ${ }_{10 \text { s. }}$ Transfection was recognized as red (Syp-RFP) branches. Red and green squares show examples of stimulation-induced fluo2 2 puncta with and without transfection, respectively. Right, Left and middle columns superimposed. G, Fluo2 fluorescence raw data (left, a.u.) and fractional changes (right, $\Delta F / F$, fluorescence change divided by the mean of baseline fluorescence) induced by Train ${ }_{10 \text { s }}$ in control boutons ( $n=6$ experiments, black) and in boutons transfected with SNAP25 shRNA and Syp-RFP ( $n=6$ experiments, red). $\boldsymbol{H}$, Antibody staining against RFP (recognizing Syp-RFP, left) and synapsin (middle, bouton marker, green) in a culture transfected with SNAP25 shRNA and Syp-RFP. Right, Left and middle panels superimposed.
} docytosis by the same molecules, SNAP25 and synaptobrevin, may provide a solution to this puzzle. If the same molecules that are involved in exocytosis are also involved in controlling subsequent endocytosis, changing the exocytosis amount changes the number of molecules involved in endocytosis, which may change the amount of endocytosis to match the exocytosis amount.

The molecular mechanisms by which SNARE proteins couple exocytosis to endocytosis is unclear. Binding studies provide some clues. For example, SNAP25 binds to an endocytic protein intersectin (Okamoto et al., 1999). The N-terminal half of the SNARE motif of synaptobrevin binds to the ANTH domain of endocytic adaptors AP180 and clathrin assembly lymphoid myeloid leukemia (CALM) (Koo et al., 2011; Miller et al., 2011), both of which may be involved in endocytosis (Zhang et al., 1998; Dittman and Ryan, 2009). Syntaxin binds dynamin, a GTPase that mediates vesicle fission, raising the possibility that syntaxin may also be involved endocytosis (Galas et al., 2000). We did not examine this possibility because we were unable to efficiently knock down both syntaxin $1 \mathrm{a}$ and $1 \mathrm{~b}$ isoforms. After knockdown of SNAP25 or synaptobrevin, it remains possible that other SNARE isoforms participate in endocytosis, but with a lower efficiency, causing the slowdown of endocytosis. This scenario is 
A

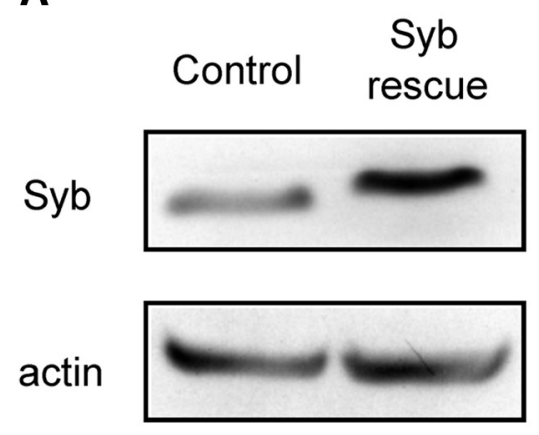

D

Control (SypH2X + scrambled shRNA)

GFP

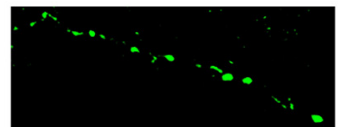

GFP

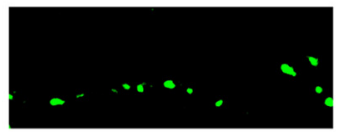

$$
\text { GFP }
$$

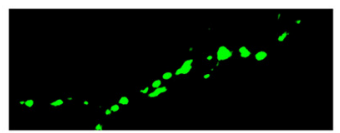

GFP

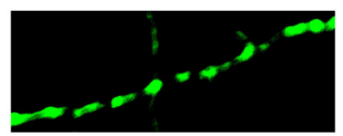

Clathrin

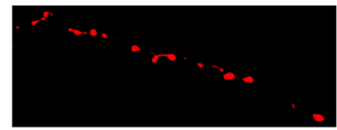

Dynamin

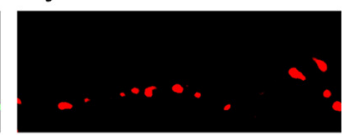

AP2

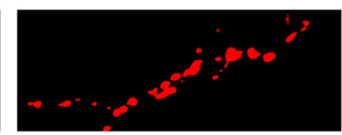

Endophilin

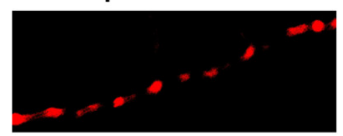

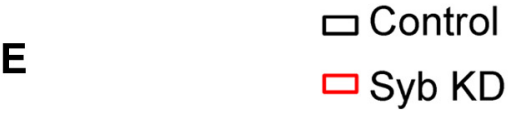

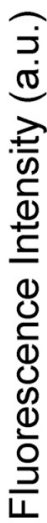

B GFP

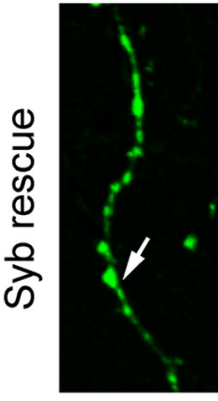

Syb

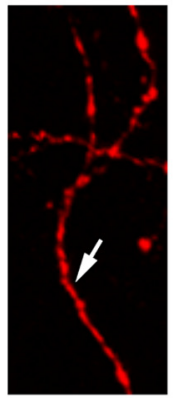

overlay

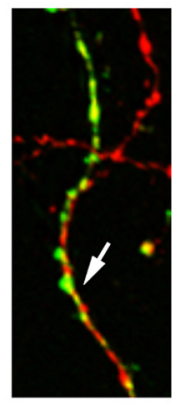

$\overline{5 \mu \mathrm{m}}$
C

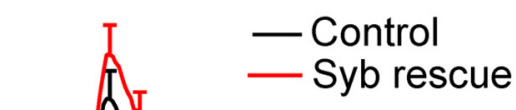

- Syb rescue

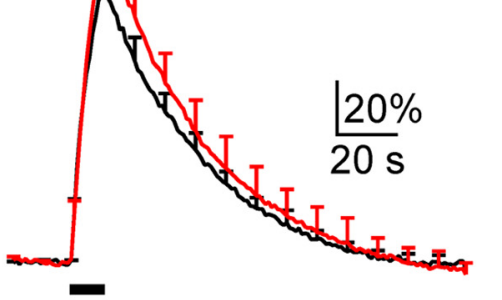

Train $_{10 \mathrm{~s}}$

Syb KD (SypH2X + shRNA)

GFP

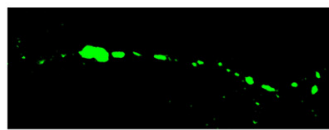

GFP

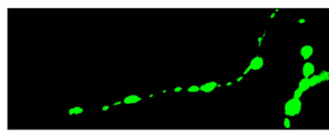

GFP

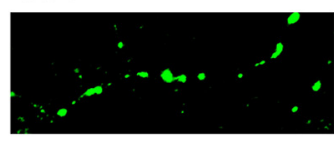

GFP

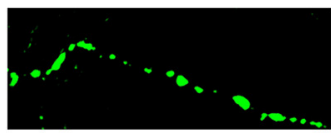

$\mathbf{F}$

- Control

- Syb KD
Clathrin

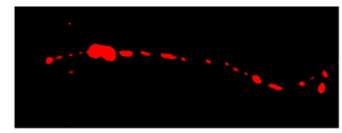

Dynamin

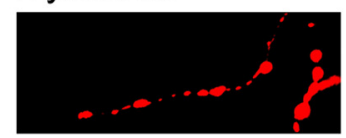

AP2

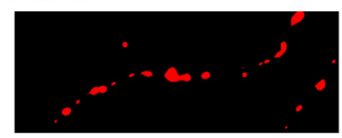

Endophilin

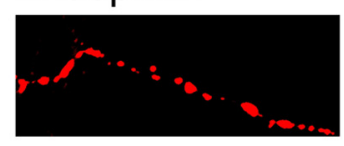

$5 \mu \mathrm{m}$
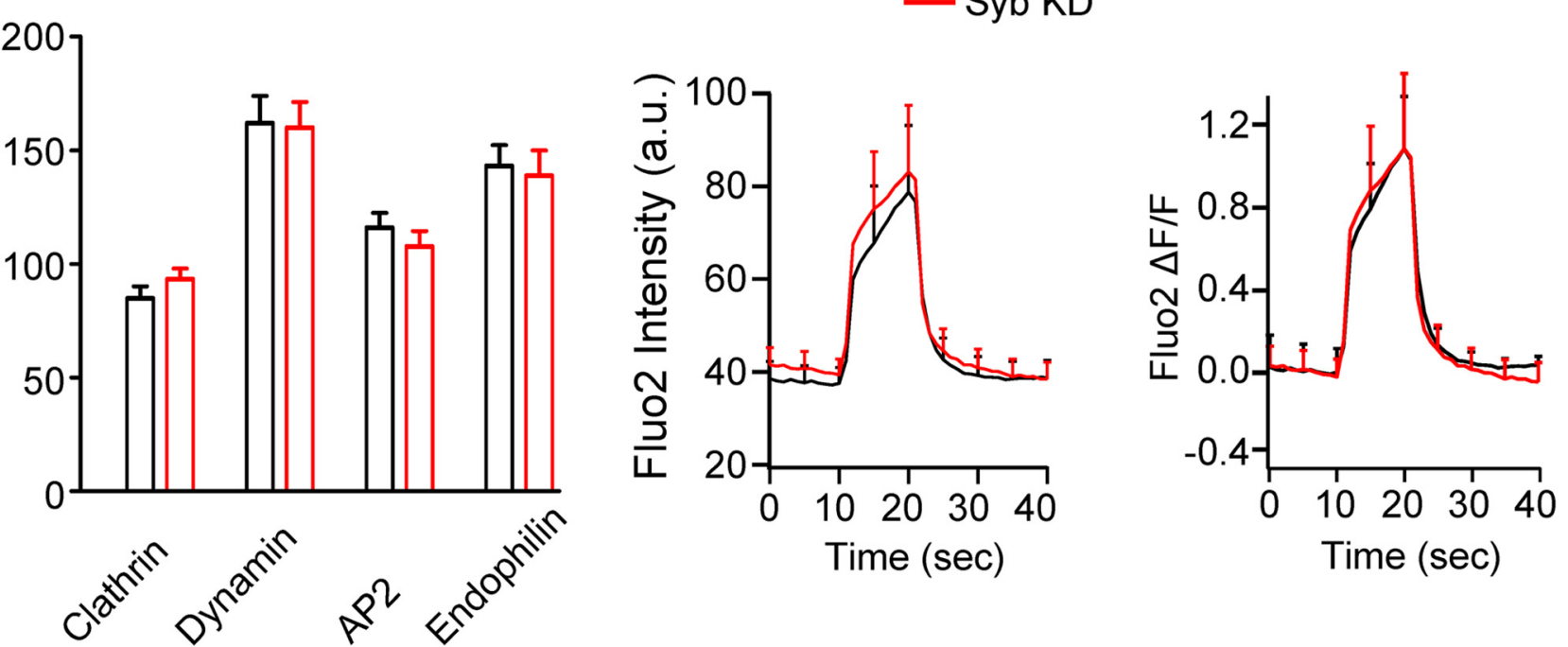

Figure 4. Synaptobrevin knockdown is specific. The arrangements in $A-E$ and $\boldsymbol{F}$ are the same as Figure $2 A-E$ and Figure $2 G$, respectively, except that SNAP25 in all legends and panels is replaced with synaptobrevin (or Syb). $\boldsymbol{C}$, Control, $n=6$ experiments; Syb rescue, $n=6$ experiments). $\boldsymbol{E}$, Each group was taken from 2 transfections (clathrin: control, $n=125$ boutons; KD, $n=141$ boutons; dynamin: control, $n=110$ boutons; KD, $n=134$ boutons; AP2: control, $n=106$ boutons; KD, $n=95$ boutons; endophilin: control, $n=120$ boutons; KD, $n=128$ boutons). $F$, control, $n=6$ experiments; Syb KD, $n=6$ experiments. 
consistent with our conclusion that SNAP25 and synaptobrevin are involved in endocytosis.

Our finding is apparently different from a study suggesting no involvement of SNAP25 in endocytosis, which is based on the observation that SNAP25 knock-out does not affect FM dye uptake into hippocampal boutons after sucrose application (Bronk et al., 2007). While the conclusion is different, the original data are not, because we used action potential stimulation to induce exocytosis and endocytosis, whereas they used sucrose because of the lack of release during action potential trains in SNAP25knock-out boutons (Bronk et al., 2007). The nature of sucroseinduced exocytosis and endocytosis is largely unclear. Sucrose induces calcium-independent exocytosis (Rosenmund and Stevens, 1996), whereas depolarization during physiological action potential trains induces calcium-dependent exocytosis. Sucroseloaded FM dye cannot be released by depolarization (Bronk et al., 2007), whereas depolarization-loaded FM dye can be rereleased by depolarization (Ryan et al., 1993; Ryan et al., 1996). It is therefore possible that sucrose-induced endocytosis is different from endocytosis induced by physiological action potentials. In SNAP25-knock-out synapses, depolarization induced by high potassium application induced a low rate of release, but no HRP or FM dye uptake (Bronk et al., 2007). This result is consistent with the involvement of SNAP25 in depolarization-induced endocytosis, although release might be too low to draw a reliable conclusion (Bronk et al., 2007). We concluded that in physiological action potential stimulation, SNAP25 is involved in endocytosis.

A recent study showed that tetanus toxin blocks slow endocytosis at a giant nerve terminal, the calyx of Held, suggesting the involvement of synaptobrevin in slow endocytosis (Hosoi et al., 2009). The present work consolidates this finding by using knockdown techniques to exclude the possibility that endocytosis blocked by tetanus toxin was a side effect caused by tetanus toxincleaved synaptobrevin fragments. Furthermore, the present work extends this earlier finding from the giant calyx nerve terminal to a conventional small bouton, and from the involvement of only synaptobrevin to the involvement of both synaptobrevin and SNAP25 in endocytosis. Together, the dual role of SNARE proteins in both exocytosis and endocytosis may be a general property of the synapse.

\section{References}

Balaji J, Armbruster M, Ryan TA (2008) Calcium control of endocytic capacity at a CNS synapse. J Neurosci 28:6742-6749. CrossRef Medline

Bronk P, Deák F, Wilson MC, Liu X, Südhof TC, Kavalali ET (2007) Differential effects of SNAP-25 deletion on $\mathrm{Ca}^{2+}$-dependent and $\mathrm{Ca}^{2+}$ independent neurotransmission. J Neurophysiol 98:794-806. CrossRef Medline

Deák F, Schoch S, Liu X, Südhof TC, Kavalali ET (2004) Synaptobrevin is essential for fast synaptic-vesicle endocytosis. Nat Cell Biol 6:1102-1108. CrossRef Medline

Dittman J, Ryan TA (2009) Molecular circuitry of endocytosis at nerve terminals. Ann Rev Cell Dev Biol 25:133-160. CrossRef Medline

Galas MC, Chasserot-Golaz S, Dirrig-Grosch S, Bader MF (2000) Presence of dynamin-syntaxin complexes associated with secretory granules in adrenal chromaffin cells. J Neurochem 75:1511-1519. Medline

Granseth B, Odermatt B, Royle SJ, Lagnado L (2006) Clathrin-mediated endocytosis is the dominant mechanism of vesicle retrieval at hippocampal synapses. Neuron 51:773-786. CrossRef Medline

Granseth B, Odermatt B, Royle SJ, Lagnado L (2009) Comment on "The dynamic control of kiss-and-run and vesicular reuse probed with single nanoparticles". Science 325:1499; author reply 1499. CrossRef Medline

He L, Wu LG (2007) The debate on the kiss-and-run fusion at synapses. Trends Neurosci 30:447-455. CrossRef Medline

Holderith N, Lorincz A, Katona G, Rózsa B, Kulik A, Watanabe M, Nusser Z
(2012) Release probability of hippocampal glutamatergic terminals scales with the size of the active zone. Nat Neurosci 15:988-997. CrossRef Medline

Hosoi N, Holt M, Sakaba T (2009) Calcium dependence of exo- and endocytotic coupling at a glutamatergic synapse. Neuron 63:216-229. CrossRef Medline

Jackson MB, Chapman ER (2008) The fusion pores of $\mathrm{Ca}^{2+}$-triggered exocytosis. Nat Struct Mol Biol 15:684-689. CrossRef Medline

Koo SJ, Markovic S, Puchkov D, Mahrenholz CC, Beceren-Braun F, Maritzen T, Dernedde J, Volkmer R, Oschkinat H, Haucke V (2011) SNARE motif-mediated sorting of synaptobrevin by the endocytic adaptors clathrin assembly lymphoid myeloid leukemia (CALM) and AP180 at synapses. Proc Natl Acad Sci U S A 108:13540-13545. CrossRef Medline

Leitz J, Kavalali ET (2011) Ca(2)(+) influx slows single synaptic vesicle endocytosis. J Neurosci 31:16318-16326. CrossRef Medline

Miller SE, Sahlender DA, Graham SC, Höning S, Robinson MS, Peden AA, Owen DJ (2011) The molecular basis for the endocytosis of small R-SNAREs by the clathrin adaptor CALM. Cell 147:1118-1131. CrossRef Medline

Okamoto M, Schoch S, Südhof TC (1999) EHSH1/intersectin, a protein that contains $\mathrm{EH}$ and $\mathrm{SH} 3$ domains and binds to dynamin and SNAP-25. A protein connection between exocytosis and endocytosis? J Biol Chem 274:18446-18454. CrossRef Medline

Prodanov D, Heeroma J, Marani E (2006) Automatic morphometry of synaptic boutons of cultured cells using granulometric analysis of digital images. J Neurosci Methods 151:168-177. CrossRef Medline

Renden R, von Gersdorff H (2007) Synaptic vesicle endocytosis at a CNS nerve terminal: faster kinetics at physiological temperatures and increased endocytotic capacity during maturation. J Neurophysiol 98:3349-3359. CrossRef Medline

Rosenmund C, Stevens CF (1996) Definition of the readily releasable pool of vesicles at hippocampal synapses. Neuron 16:1197-1207. CrossRef Medline

Ryan TA, Reuter H, Wendland B, Schweizer FE, Tsien RW, Smith SJ (1993) The kinetics of synaptic vesicle recycling measured at single presynaptic boutons. Neuron 11:713-724. CrossRef Medline

Ryan TA, Smith SJ, Reuter H (1996) The timing of synaptic vesicle endocytosis. Proc Natl Acad Sci U S A 93:5567-5571. CrossRef Medline

Sankaranarayanan S, Ryan TA (2001) Calcium accelerates endocytosis of vSNARE at hippocampal synapses. Nat Neurosci 4:129-136. CrossRef Medline

Sheng J, He L, Zheng H, Xue L, Luo F, Shin W, Sun T, Kuner T, Yue DT, Wu LG (2012) Calcium-channel number critically influences synaptic strength and plasticity at the active zone. Nat Neurosci 15:998-1006. CrossRef Medline

Smith C, Neher E (1997) Multiple forms of endocytosis in bovine adrenal chromaffin cells. J Cell Biol 139:885-894. CrossRef Medline

Sudhof TC (2004) The synaptic vesicle cycle. Annu Rev Neurosci 27: 509-547. CrossRef Medline

Sun JY, Wu XS, Wu LG (2002) Single and multiple vesicle fusion induce different rates of endocytosis at a central synapse. Nature 417:555-559. CrossRef Medline

Sun T, Wu XS, Xu J, McNeil BD, Pang ZP, Yang W, Bai L, Qadri S, Molkentin JD, Yue DT, Wu LG (2010) The role of calcium/calmodulin-activated calcineurin in rapid and slow endocytosis at central synapses. J Neurosci 30:11838-11847. CrossRef Medline

Wu LG, Betz WJ (1996) Nerve activity but not intracellular calcium determines the time course of endocytosis at the frog neuromuscular junction. Neuron 17:769-779. CrossRef Medline

Wu LG, Ryan TA, Lagnado L (2007) Modes of vesicle retrieval at ribbon synapses, calyx-type synapses, and small central synapses. J Neurosci 27: 11793-11802. CrossRef Medline

Zhang B, Koh YH, Beckstead RB, Budnik V, Ganetzky B, Bellen HJ (1998) Synaptic vesicle size and number are regulated by a clathrin adaptor protein required for endocytosis. Neuron 21:1465-1475. CrossRef Medline

Zhang Q, Li Y, Tsien RW (2009) The dynamic control of kiss-and-run and vesicular reuse probed with single nanoparticles. Science 323:1448-1453. CrossRef Medline

Zhu Y, Xu J, Heinemann SF (2009) Two pathways of synaptic vesicle retrieval revealed by single-vesicle imaging. Neuron 61:397-411. CrossRef Medline 